\title{
Generalized Transition Probability, Mobility and Symmetries
}

\author{
Vittorio Cantoni \\ Istituto Matematico dell'Università, via Saldinı 50, I-20133 Milano, Italy
}

\begin{abstract}
In the framework of Mackey's description of a physical system, the generalized transition probability, as defined in an earlier paper, is shown to be non-decreasing while the system evolves, and invariant when the evolution is reversible. It is also invariant under a natural action of the space-time symmetry group.
\end{abstract}

\section{Introduction}

As in [1], we shall adopt Mackey's description of a physical system in terms of a set $\mathscr{S}$ of states, a set $\mathcal{O}$ of observables, and a structure function $\not p$ representing the probability distributions associated with the measurements of the observables on the states [2]. This broad framework is enough to define a generalized transition probability $T(\alpha, \beta)$ on $\mathscr{S} \times \mathscr{S}$ (or, equivalently, a distance function $d(\alpha, \beta)$ which turns $\mathscr{S}$ into a metric space). [1, 3-5].

Additional structure must be specified in order to exhibit how the generalized transition probability is related to the dynamical evolution of the system, and to the space-time symmetry group in a relativistic theory.

Following Mielnik $[6,7]$, we shall represent the set of all possible evolutions of the physical system by a mobility semigroup $\mathscr{M}$. Its natural action on $\mathscr{S}$ and $\mathcal{O}$ will turn out to be such that the generalized transition probability between any pair of evolving states cannot decrease with time. Under an additional reversibility assumption, $\mathscr{M}$ becomes a group and $T$ is preserved. Similarly, in a relativistic theory, the space-time symmetry group $\mathscr{G}$ must have a natural action on $\mathscr{S}$ and $\mathcal{O}$ such that the function $T$ be preserved.

We shall conclude by remarking that, in this perspective, the purely metric aspects of the quantum-mechanical formalism, directly related to observation via the transition probability, acquire a primary significance, while the underlying linear structure, and all its important consequences, appear in a certain sense as derived elements - a remark which seems pertinent both to the justification of the established formalism and to the search for its possible extensions or modifications. 


\section{Mackey Systems and Mobility}

Recall that, in Mackey's scheme, the function $\not(A, \alpha, E)$ is interpreted as the probability that a measurement of the observable $A \in \mathcal{O}$ on the state $\alpha \in \mathscr{S}$ give a result in the Borel set $E$ of the real line $\mathrm{R}$. Thus to any given pair $(A, \alpha)$ there corresponds a probability measure $\alpha_{A}$ on $\mathrm{R}$ such that $\int_{E} d \alpha_{A}=\not(A, \alpha, E)$. If $\beta$ is a second state, one can consider the number

$$
T_{A}(\alpha, \beta)=\left|\int_{R} d \sqrt{\alpha_{A} \beta_{A}}\right|^{2}
$$

where $\sqrt{\alpha_{A} \beta_{A}}$ is the Kakutani product of the measures $\alpha_{A}$ and $\beta_{A}[8]$, and define the generalized transition probability $T$ between $\alpha$ and $\beta$ by setting

$$
T(\alpha, \beta)=\inf _{A \in \mathscr{O}} T_{A}(\alpha, \beta)
$$

The set $\mathscr{S}$ becomes a metric space if the distance function $d$ is defined by the relation

$$
d(\alpha, \beta)=\sqrt{2\left[1-T^{1 / 2}(\alpha, \beta)\right]} .
$$

The knowledge of $d$ is completely equivalent to the knowledge of $T$.

Each state $\alpha$ represents a class of equivalent "preparations," and must therefore correspond to a "reference time" $t$, which can conveniently be thought of as the instant at which the preparation is completed. An ensemble associated with $\alpha$ is obtained by repeating the same preparation as many times as one wishes under identical conditions, at reference times $t^{\prime}, t^{\prime \prime}, \ldots$ Similarly, each observable $A$ represents a class of equivalent measurements, and must therefore correspond to a "reference time" $t$, which can now conveniently be thought of as the instant at which the measurement begins. The measurement of $A$ can be repeated as many times as one wishes, at reference times $t^{\prime}, t^{\prime \prime}, \ldots$; its individual outcomes are in general variable (even when they follow identical preparations), but the statistical distributions are the same on ensembles associated with the same state.

Now suppose that, having performed a preparation of $\alpha$ at reference time $t$, one lets the system evolve during a time-interval $(t, t+\tau)$ under fixed, controlled environmental conditions, and then performs the measurement of $A$ at reference time $t+\tau$. Such an experiment (which can in principle be repeated as many times as one wishes) can be interpreted in two ways: as the preparation of the state $\alpha$ (at reference time $t$ ) followed by the measurement of a new observable $m A$ (at reference time $t$ ), or as the preparation of a new state $m \alpha$ (at reference time $t+\tau$ ) followed by the measurement of $A$ (at reference time $t+\tau$ ). The correspondences $m: A \rightarrow m A$ and $m: \alpha \rightarrow m \alpha$ are completely determined by $\tau$ and by the selected external conditions during the interval $(t, t+\tau): m \alpha$ is prepared by performing the preparation of $\alpha$ and letting the system evolve under the selected conditions; the observable $m A$ is measured by applying the selected conditions and measuring $A$ with a delay $\tau$.

By letting $\tau$ and the environmental conditions during the time-interval $(t, t+\tau)$ vary in all physically realizable ways, one obtains the set $\mathscr{M}$ of all possible movements of the system. Each movement $m \in \mathscr{M}$ determines, as we have just seen, a 
correspondence $\alpha \rightarrow m \alpha$ from $\mathscr{S}$ to $\mathscr{P}$ as well as a correspondence $A \rightarrow m A$ from $\mathcal{O}$ to $\mathcal{O}$. If, as we shall assume, any two choices of external conditions (with definite durations) can be applied in immediate succession, one has a natural composition of movements which determines a semigroup structure in $\mathscr{M}$.

The actions of the elements of $\mathscr{M}$ on $\mathscr{S}$ and on $\mathcal{O}$ are such that the relation

$$
\not(A, m \alpha, E)=\not(m A, \alpha, E)
$$

is identically satisfied, since, for any fixed choice of $A, \alpha$ and $m$, the two statistical distributions corresponding to the left and right-hand sides of (4) are determined by the same set of experiments.

The above considerations suggest the adjunction, to Mackey's scheme consisting of the triple $\{\mathscr{S}, \mathcal{O}, \not\}$, of a mobility semigroup $\mathscr{M}$. In addition to Mackey's axioms I and II ([2] p. 62) ${ }^{1}$ an action of the semigroup $\mathscr{M}$ on $\mathscr{S}$ and on $\mathcal{O}$ satisfying the identity (4) should be assumed.

Note that, although we have adopted in part Mielnik's concepts and terminology, at the present stage we neither make the assumption that the maps by which $\mathscr{M}$ acts on $\mathscr{S}$ be invertible, nor do we require that $\mathscr{S}$ be a manifold (compare with [6] and [7]).

\section{Dynamical Behaviour of the Generalized Transition Probability}

From the definition (1) and the identity (4), one immediately obtains the relation

$$
T_{A}(m \alpha, m \beta)=T_{m A}(\alpha, \beta) .
$$

Since there is no a priori reason to assume that the maps $m: \mathcal{O} \rightarrow \mathcal{O}$ be surjective, one has $m O \subset O$ (every "delayed observable" is an observable) but not necessarily $m \mathcal{O}=\mathcal{O}$ (some observables may not be equivalent to "delayed" ones). By considering the greatest lower bounds of the left and right-hand sides of (5), one therefore gets, remembering the definition (2) of $T$ :

$$
\begin{aligned}
T(m \alpha, m \beta) & =\inf _{A \in \mathcal{O}} T_{A}(m \alpha, m \beta)=\inf _{m A \in m \mathcal{O}} T_{m A}(\alpha, \beta) \\
& =\inf _{A \in m \mathcal{O}} T_{A}(\alpha, \beta) \geqq \inf _{A \in \mathcal{O}} T_{A}(\alpha, \beta)=T(\alpha, \beta) .
\end{aligned}
$$

Thus each movement $m \in \mathscr{M}$ must be represented on $\mathscr{S}$ by a map $m: \mathscr{S} \rightarrow \mathscr{S}$ such that the function $T$ is non-decreasing

$$
T(\alpha, \beta) \leqq T(m \alpha, m \beta)
$$

Axiom $I: \quad \not(A, \alpha, \phi)=0, \not(A, \alpha, R)=1$;

$$
\not\left(A, \alpha, E_{1} \cup E_{2} \cup \ldots\right)=\sum_{j} \not\left(A, \alpha, E_{j}\right) \text { whenever }
$$

the Borel sets $E_{j}$ are disjoint in pairs.

Axiom II: $\not(A, \alpha, E)=\not\left(A^{\prime}, \alpha, E\right)$ for all $\alpha$ and $E \Rightarrow A=A^{\prime}$; $\not(A, \alpha, E)=\not\left(A, \alpha^{\prime}, E\right)$ for all $A$ and $E \Rightarrow \alpha=\alpha^{\prime}$ 
Equivalently, the distance function $d$ must be non-increasing:

$$
d(\alpha, \beta) \geqq d(m \alpha, m \beta) .
$$

If the actual time-evolution of the physical system is represented by a oneparameter family of movements, the generalized transition probability between any pair of evolving states corresponding to fixed initial conditions must be a nondecreasing function of time. Equivalently, the distance function must be a nonincreasing function of time.

\section{Reversible Systems}

Certain restrictions on the behaviour of the physical system imply that relations (7) and (8) are always satisfied, for such systems, with the equality sign. This happens, in particular, if each movement $m \in \mathscr{M}$ admits an inverse $m^{-1}$ (physically: if any arrangement of external conditions could be followed by a second arrangement restoring the initial situation). When this is the case, $\mathscr{M}$ is a group, and we shall say that the system is reversible.

Thus, with a reversible system there is associated a mobility group which preserves the generalized transition probability, and therefore acts on $\mathscr{S}$ isometrically. Moreover, one can assume that the action of $\mathscr{M}$ on $\mathscr{S}$ be transitive, since, when reversibility holds, the condition that any state should be transformable (by an adequate choice of external conditions) into any other state can be regarded as a sound operational criterion to assert that the two states belong to the same physical system.

\section{Space-Time Symmetries}

Suppose now that the physical system possesses a space-time symmetry group, in the sense that the existence of a class of physically equivalent reference frames is admitted, together with the possibility of transferring every apparatus for the preparation of the states, the measurement of the observables and the control of the external conditions from any one to any other frame of the class. Then there is a natural action of the symmetry group $\mathscr{G}$ on $\mathcal{O}$ and on $\mathscr{S}$. Each element $g \in \mathscr{G}$ determines the bijections $g: \mathscr{S} \rightarrow \mathscr{S}, \alpha \rightarrow g \alpha$ and $g: \mathcal{O} \rightarrow \mathcal{O}, A \rightarrow g A$, and the physical equivalence of the reference frames entails the relation

$$
\not h(A, \alpha, E)=\not h(g A, g \alpha, E) .
$$

Hence, recalling the definitions (2) and (3), one obtains

$$
T_{A}(\alpha, \beta)=T_{g A}(g \alpha, g \beta)
$$

and

$$
\begin{aligned}
T(\alpha, \beta) & =\inf _{A \in \mathcal{O}} T_{A}(\alpha, \beta)=\inf _{g A \in \mathcal{A} \mathcal{O}} T_{g A}(g \alpha, g \beta) \\
& =\inf _{A \in \mathcal{O}} T_{A}(g \alpha, g \beta)=T(g \alpha, g \beta),
\end{aligned}
$$


where the bijective character of the map $g: \mathcal{O} \rightarrow \mathcal{O}$ has been taken into account.

Thus, the space-time symmetry group $\mathscr{G}$ can be brought into Mackey's scheme by specifying its actions on $\mathscr{S}$ and on $\mathcal{O}$. Equation (9) must always be satisfied; consequently, the action of $\mathscr{G}$ on $\mathscr{S}$ must preserve the generalized transition probability (equivalently: it must be isometric).

\section{Remarks on the Quantum-Mechanical Formalism}

We conclude by a brief sketch of arguments which support the view that the significant structures involved in the quantum-mechanical model are directly related to the transition probability function and its symmetry group-the metric aspects of the theory - while the technically important but perhaps not conceptually indispensable reliance of the formalism on the linear structure of an underlying Hilbert space appears to be present, in this particular model, owing to the peculiar structure of the set of pure states as a metric space.

a) Being the projective space associated with a complex separable Hilbert space $\mathscr{H}$, the set of pure states $\mathscr{\mathscr { H }}$ of Quantum Mechanics is a manifold modeled on a separable Hilbert space, with a strong riemannian structure $\mathfrak{g}$ (the Fubini-Study metric, [9] p. 160, or its straightforward generalization to the infinite-dimensional case). The metric tensor $g$ can be derived from the transition probability by the procedure described in [10].

Besides $\mathfrak{g}, \tilde{\mathscr{P}}$ has a second physically relevant geometric structure: a strong symplectic form $\omega$, whose role in Quantum Mechanics has been made explicit in [11]. But $\omega$ is itself completely determined (up to a physically irrelevant change of sign) by the transition probability via the riemannian structure: in fact, the Riemann tensor of $\tilde{\mathscr{H}}$ has the form

$$
\begin{aligned}
R(W, X, Y, Z)= & \mathfrak{g}(W, Z) \mathfrak{g}(X, Y)-\mathfrak{g}(W, Y) \mathfrak{g}(X, Z)+\omega(W, Z) \omega(X, Y) \\
& -\omega(W, Y) \omega(X, Z)-2 \omega(W, X) \omega(Y, Z)
\end{aligned}
$$

(where $W, X, Y, Z$ are vectors tangent to $\tilde{\mathscr{H}}$ ), from which one immediately derives the relation

$$
3[\omega(X, Y)]^{2}=[\mathfrak{g}(X, Y)]^{2}-\mathfrak{g}(X, X) \mathfrak{g}(Y, Y)-R(X, Y, X, Y),
$$

which demonstrates the assertion.

Thus the entire intrinsic geometric structure of $\widetilde{\mathscr{H}}$ (Kähler manifold with constant and positive holomorphic sectional curvature) is determined by the transition probability.

b) In Quantum Mechanics each observable $A$ is represented by a self-adjoint operator $\mathbf{A}$ of $\mathscr{H}$. If $\mathbf{A}$ and $\mathbf{A}^{\prime}$ only differ by a multiple of the identity, the observables $A$ and $A^{\prime}$ describe essentially the same physical quantity.

Equivalently, each physical quantity can be represented by the one-parameter group of unitary transformations (determined up to a factor of modulus one) generated by any of its self-adjoint representatives. Thus each physical quantity is associated with a well-determined one-parameter group of isometries of $\tilde{\mathscr{H}}$ (regarded as a metric space), unaffected by a change of the representative (since 
multiplication by a complex number of modulus 1 in $\mathscr{H}$ does not affect the transition probability).

Such a representation of the physical quantities by means of one-parameter groups of isometries of the state space makes use of metric concepts only, and does not rely on the linear structure of $\mathscr{H}$. It would make sense in any space $\mathscr{S}$ with a transition probability admitting a nontrivial symmetry group, even if $\mathscr{S}$ were not a projective space.

The same is true for the quantum commutation relations. If two quantities are represented by the operators $\mathbf{A}$ and $\mathbf{B}$ and by the one-parameter subgroups $U_{\lambda}^{A}$ and $U_{\lambda}^{B}$ of isometries of $\tilde{\mathscr{H}}$, their quantum commutator $[A, B]$ is represented by the operator $i / \hbar(\mathbf{A B}-\mathbf{B A})$, or by the subgroup

$$
U_{\lambda}^{[A, B]}=\exp \left[\lambda\left(\frac{d}{d \mu} U_{\sqrt{\hbar \mu}}^{A} U_{\sqrt{\hbar \mu}}^{B} U_{-\sqrt{\hbar \mu}}^{A} U_{-\sqrt{\hbar \mu}}^{B}\right)_{\mu=0}\right] .
$$

The last relation makes sense with no reference to the linear structure of $H$, and therefore lends itself to generalization.

c) In this perspective, the irreducible unitary representations "up to a factor" of the inhomogeneous Lorentz group L, described by Wigner [12] as a basis for the description of elementary systems, can also be regarded as "metric" representations, acting isometrically on the space $S$ of pure states, with no reference to the underlying linear space.

The fact that $L$ should act isometrically on $S$ in a relativistic theory has been justified in Sect. 5. The recovery of the non-relativistic quantum conditions can then be achieved by means of a suitable deformation of the Lie algebra of $L$ (corresponding to the limit $c \rightarrow \infty$ ), as recently shown by G. Kaiser [13]. This provides a promising approach to a justification of the quantum-mechanical formalism, possibly on the basis of requirements of relativistic invariance only.

\section{References}

1. Cantoni, V.: Commun. Math. Phys. 44, 125-128 (1975)

2. Mackey, G. W.: Mathematical foundations of quantum mechanics. New York: Benjamin 1963

3. Gudder, S.: Commun. Math. Phys. 63, 265-267 (1978)

4. Gudder, S.: Stochastic methods in quantum mechanics. New York, Oxford: North Holland 1979

5. Hadjisavvas, N.: Commun. Math. Phys. 83, 43-48 (1982)

6. Mielnik, B.: Rep. Math. Phys. 12, 331-339 (1977)

7. Mielnik, B.: J. Math. Phys. 21, 44-53 (1980)

8. Xia, D. X.: Measure and integration theory on infinite-dimensional spaces. New York, San Francisco, London: Academic Press 1971

9. Kobayashi, S., Nomizu, K.: Foundations of differential geometry, Vol. II. New York, Chichester, Brisbane, Toronto: John Wiley \& Sons 1969

10. Cantoni, V.: Commun. Math. Phys. 56, 189-193 (1977)

11. Cantoni, V.: Rend. Accad. Naz. Lincei 62, 628-636 (1977)

12. Wigner, E.: Ann. Math. 40, 149-204 (1939)

13. Kaiser, G.: J. Math. Phys. 22 705-714 (1981)

14. Raggio, G. A. Lett. Math. Phys. 6, 233-236 (1982)

15. Araki, H., Raggio, G. A., Lett. Math. Phys. 6, 237-240 (1982)

Communicated by R. Haag

Received April 6, 1982 